\title{
Plane surface lapping technological processor
}

\author{
Andrea Deaconescu ${ }^{1}$, and Tudor Deaconescu ${ }^{1, *}$ \\ ${ }^{1}$ Transilvania University of Braşov, Department of Industrial Engineering and Management, 500036, \\ Braşov, 29 Eroilor Bd., Romania
}

\begin{abstract}
To date lapping as a finishing process of part surfaces lacks an underlying digital knowledge base. This is due to the complex interlinking of the numerous parameters that influence the results of machining. Starting from here the paper argues improved process planning and machining process optimization by presenting a new modelling algorithm of lapping and a software tool designed as a support for lapping machine operators. The proposed computer programme enables automated planning of machining and can be deployed for studies related to the influence of various work parameters on the quality criteria targeted by machining.
\end{abstract}

\section{Introduction}

Two of the essential requirements to be met cumulatively by mechanical components machined by cutting are high form precision (very flat surfaces) and minimum surface roughness. These characteristics can be obtained by lapping, a metal cutting method obtained by the friction generated between two surfaces that are pressed one onto the other in the presence of an interfacing abrasive paste (slurry).

The applicability area of lapping practically covers all industrial branches like hydraulics, pneumatics, electrotechnics, computers, precision mechanics and optics, machines manufacturing, automotive and aircraft industry, etc. Lapping is used in the machining process of a large variety of parts, like mechanical seals, medical equipment (pacemaker components, surgical instruments), tools, measuring instruments, and lends itself particularly for the machining of reference surfaces, base and guiding surfaces, surfaces of hydraulic pump pistons, of disk drive heads, etc. Lapped surfaces are free from surface stress and benefit from high wear strength.

Lapping is strongly influenced by two groups of input quantities. The first group relates to the lapping paste (slurry) and includes the size, form, hardness and friability of the abrasive grains, grain size distribution, lapping paste concentration and carrier fluid viscosity. The second group of parameters pertains to the lapping process and includes information concerning workpiece material, size and form of the lapped surfaces, the machining allowance, machining pressure and speed, the kinematics of the lapping process, etc. The large number of work parameters to be considered calls for a clear and thorough understanding of their interlinking. At present operators face difficulties in selecting an optimum combination of all these working parameters.

\footnotetext{
*Corresponding author : tdeacon@unitbv.ro
} 
To date literature related to surface lapping does not supply general calculation formulae or relationships that link together the various parameters impacting the results of machining. The experimental values published in literature relate strictly to the concrete cases that are discussed and should not be generalized. Lapping still lacks a digital data base of set points of the input and related output values of the machining process. The availability of such would be a strong instrument for improving manufacturing in both the design and the actual machining phase.

Considering the above aspects, the paper puts forward a modelling algorithm of the plane (flat) lapping process and of a software tool designed as a support for lapping machine operators. This instrument enables the operator to determine in minimum time the set-points for the adjustable input values of the machining system such as to ensure the targeted output in terms of surface quality.

\section{Theoretical bases}

The authors conceived a dedicated programme for flat lapping (FLaT) working with application-specific algorithms and data bases and that is easily mastered by the user.

The work algorithm underlying the developed software tool start from the spherical model of an abrasive grain. This type of model was put forward by Moon who, using a scanning electron microscope (SEM) in his research demonstrated a quasi-spherical shape of grit deployed in lapping [1]. Luo and Dornfeld confirm the accuracy of process modelling underlain by a sphere shape [2]. Figure 1 presents the spherical model of a grain and the rationale underlying the development of such model.

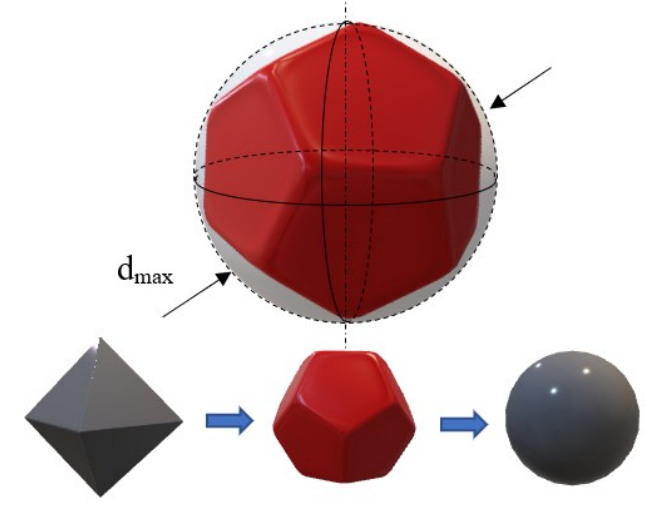

Fig. 1. Spherical approximation of the abrasive particle shape.

Lapping grit must come in an isometric (compact) shape, a requirement that is ensured by the discussed model. This prevents the orientation of the grains by certain preferential directions.

The developed software tool provides the operator of the lapping machine with information related to the quantities of interest: the duration of processing $(\mathrm{min})$, the thickness of the material cut off in the lapping process $(\mu \mathrm{m})$, the rate at that the material is cut $(\mu \mathrm{m} / \mathrm{min})$, the volume of active grit brought into the cutting area in a time unit $\left(\mathrm{mm}^{3} / \mathrm{min}\right)$ and the necessary abrasive mass $(\mathrm{g})$. The values of these quantities were determined by means of computational relationships detailed in [3].

Thus, the machining time is calculated by equation (1):

$$
t=\frac{\sqrt[v R]{R_{a f}}-\sqrt[v R]{R_{a i}}}{\sqrt[v R]{K_{R} \cdot e^{x R \cdot C^{y R} \cdot v r^{2 R} \cdot p^{u R}}}}
$$


including the following notations:

- $R_{a i}, R_{a f}$ - superficial quality parameters (roughness), prior and subsequently to machining, respectively;

- $K R, v R, x R, y R, z R, u R$ - elements intervening in equation (1) that were determined by experiment. As mentioned, FLaT works with a data base that contains the concrete values of these coefficients depending on the respective material [4];

- $e$ - offset (or eccentricity) of the lap disk and workpiece support vertical axes;

- $C$-percentage of abrasive material within the slurry;

- $v r$ - relative velocity of the lapping system;

- $p$-machining pressure.

Equation (2) computes the thickness of the material that is cut off:

$$
h=K_{h} \cdot e^{x h} \cdot C^{y h} \cdot v r^{z h} \cdot p^{u h} \cdot t^{v h}
$$

where $K_{h}, v h, x h, y h, z h, u h, v h$ are elements intervening in equation (2) that were determined by experiment and retrieved for any specific material by the software tool from the same data base [4].

Essential for determining the values of the abrasive flow rate and of the abrasive mass is an accurate prediction of the number of abrasive grains that participate in the machining process. It is known that only the large grains actually remove material from the machined surface, namely grains with dimensions larger than a certain value $\delta$ up to their maximum value $d_{\max }$. Figure 2 shows for F400 grains the normal distribution of grain size and the interval of useful grain dimension $\left[\delta ; d_{\max }\right]$.
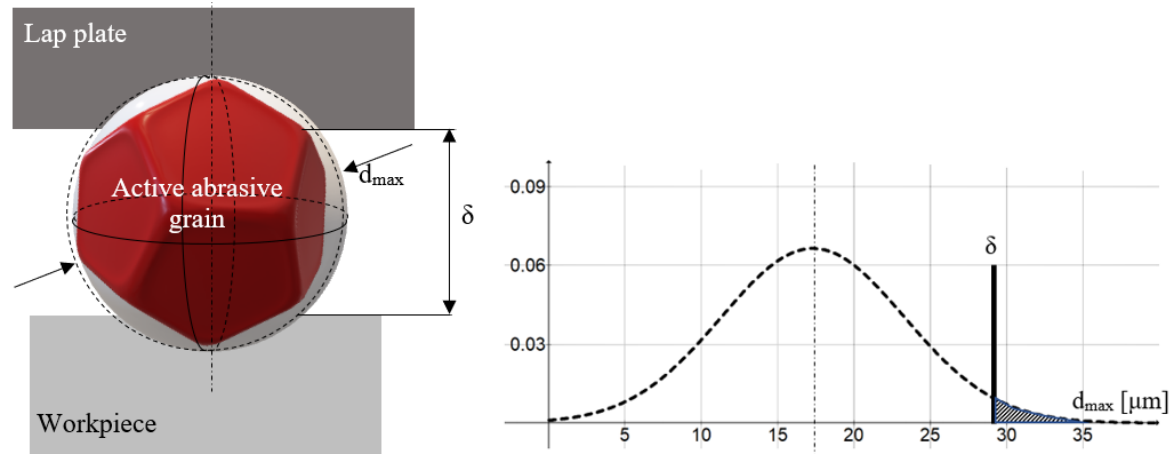

Fig. 2. Penetration of a spherical abrasive grain into the lap plate and into the machined surface and the normal distribution of grain sizes.

Equation (3) calculates the fraction of abrasive grains that actively carry out cutting (namely grains of dimension $>\delta$ ):

$$
F_{p}=0.5-\frac{1}{\sqrt{2 \cdot \pi}} \int_{0}^{z} e^{-\frac{z^{2}}{2}} \cdot d z
$$

where the variable $z$ is:

$$
z=\frac{\delta-d_{m e d}}{\sigma}
$$

whereas $d_{\text {med }}$ denotes the average grains size.

Upon denoting by $S$ the area of the machined surface and by $\rho_{l}$, and $\rho_{a}$ the density of the liquid that is used for the abrasive slurry and of the abrasive material, respectively, the 
volume of active grit brought into the cutting area in a time unit $\left(Q_{a}\right)$ and the necessary abrasive mass $\left(m_{a}\right)$ are calculated by means of equations (5) and (6), respectively:

$$
\begin{gathered}
Q_{a}=\frac{\rho_{l} \cdot C \cdot S \cdot(h+\delta)}{\rho_{a} \cdot(1-C)+\rho_{l} \cdot C} \cdot \frac{1}{100 \cdot F_{p \cdot t}} \\
m_{a}=\rho_{a} \cdot V_{a}
\end{gathered}
$$

The material removal rate $(M R R)$ is calculated by equation (7) in function of the thickness of the material layer that is cut off $(h)$ and of the time of machining $(t)$ :

$$
M R R=\frac{h}{t}
$$

These equations were used for the development of the FlaT software tool, conceived for enabling the swift programming of machining by plane lapping.

\section{FLaT - a Dedicated Technology Planning Instrument}

Planning and optimization of flat surface lapping is achieved by means of an original expert CAD-T (Computer Aided Design - Technological) system, that was developed consequently to theoretical and experimental studies. One of the components of this CAD-T system is the so-called technological processor that provides the end-user with the required technological documentation. Figure 3 shows the algorithm that underlies the technological processor:

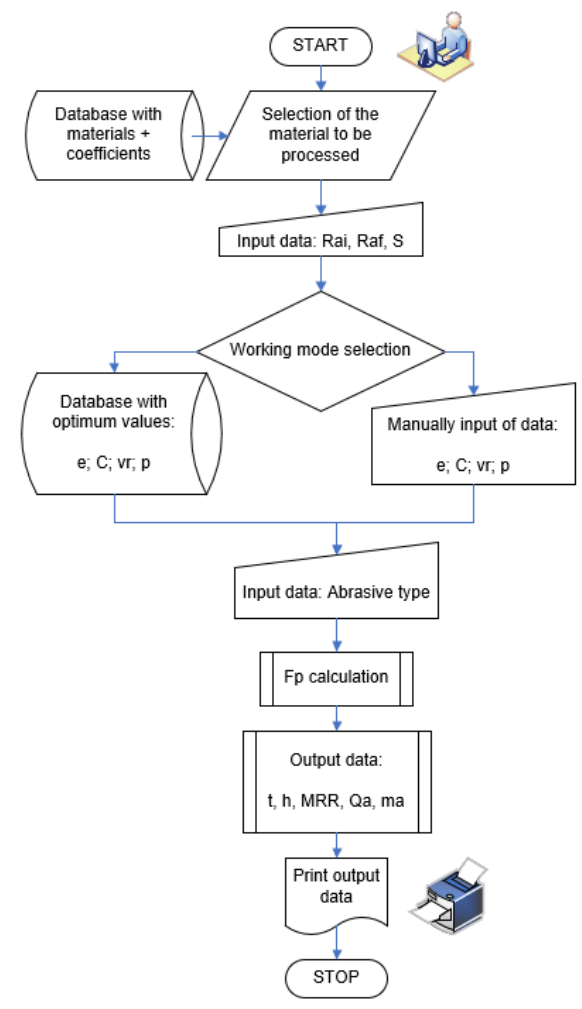

Fig. 3. Flow chart of the technological processor. 
As a first step the user choses the identifier of the steel that will be machined. A data base programmed in MS-Access is available with data on the properties of most materials typically machined by lapping, including the values of the coefficients and exponents in equations (1) and (2). The data base is permanently updated [4].

The following step requires the user to enter the magnitude of the machined surface (in $\mathrm{cm}^{2}$ ) and superficial quality parameters (roughness), prior and subsequently to machining, respectively. Subsequently two options are available: utilizing optimized input data provided by another application of the expert CAD-T system (TagMaster) or entering the currently used data manually. The optimized values provided by TagMaster are stored in another data base and by utilizing these the system undergoes robust optimisation that renders it insensitive to external disturbance factors generically known as noises [5].

In the last step the user selects the abrasive material and its graining (from another data base), upon which the application displays the results, namely the expected values of the output parameters.

Figure 4 shows the user interface of FLaT:

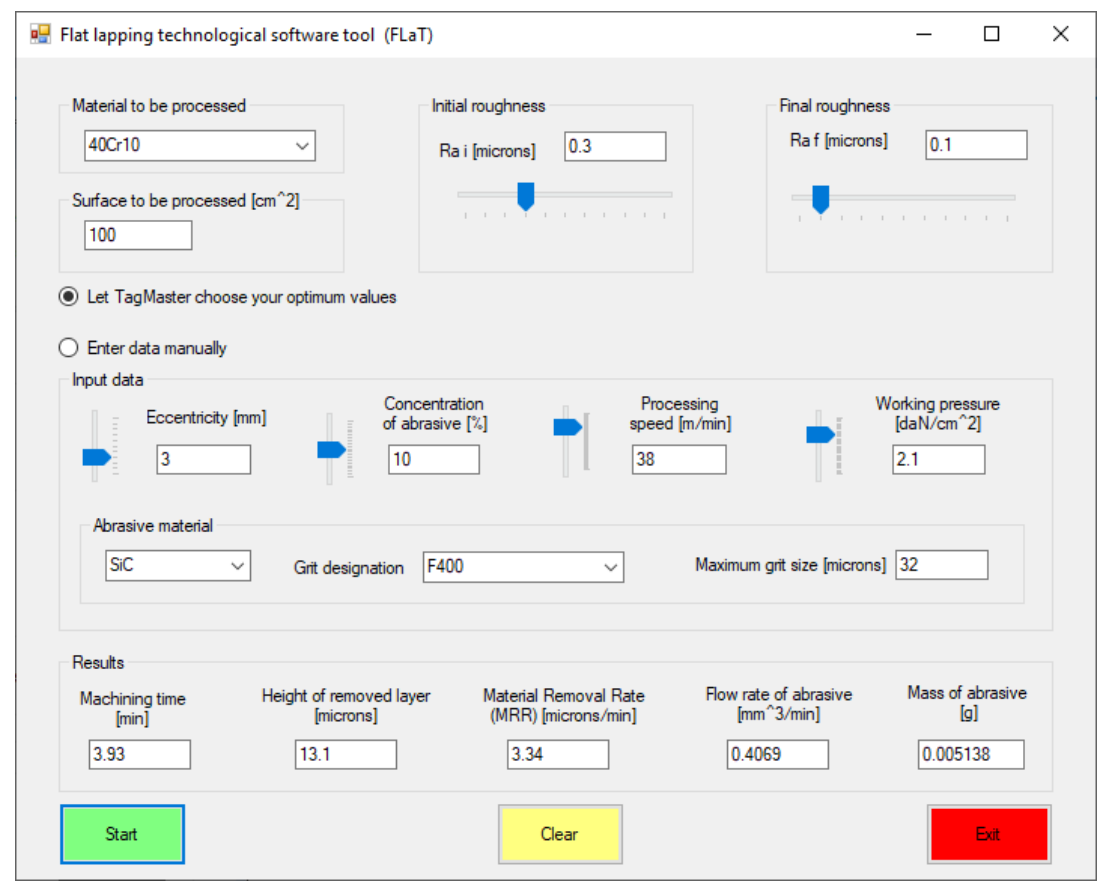

Fig. 4. User interface of the technological processor.

The deployment of the technological processor yielded a series of conclusions related to the selection of the work parameters. Thus:

- a more rapid machining is facilitated by a smaller offset and a smaller relative speed, and also when the slurry contains larger quantities of abrasive material and the work pressure is high.

- the machining allowance can be smaller when eccentricity and relative speed decrease. Modifications of work pressure and abrasive concentration do not impact significantly the thickness of the material that is cut off significantly.

- an improved material removal rate $(M R R)$ will be ensured if the slurry contains a larger quantity of abrasive material and the pressure increases. The relative speed has the smallest impact on $M R R$. 
- the flow rate of the abrasive increases for higher values of the abrasive concentration and work pressure or for smaller values of the relative speed and the eccentricity.

Tests were conducted based on the data provided by FLaT. The measured outputs in terms of surface roughness and the actually cut off material thickness met the targets and thus validated the utility of the software tool.

\section{Conclusion}

Achieving predictability of the results obtained by lapping requires extensive study of the interlinking of the many input quantities and also the development of adequate software tools. The paper presents and discusses such a tool that was developed based on an original calculation method of the lapping process. Modelling considered abrasive particles of sphere-shape and a normal distribution of dimensions.

The software application that is put forward in this paper provides the operator with the necessary technological documentation, thus significantly reducing time and effort of calculating the output quantities. In addition to its practical utility the application also offers an overview of the different impact of each of the input parameters on the outcome of machining.

\section{References}

1. Y. Moon, Mechanical aspects of the material removal mechanism in chemical mechanical polishing - CMP (Ph.D. Dissertation, University of California at Berkeley, CA, USA 1999)

2. J. Luo, D. A. Dornfeld, IEEE Trans. on semiconductor manuf., 14, 112 (2001)

3. T. Deaconescu, A. Deaconescu, Materials, 13, 1343 (2020)

4. A. Deaconescu, T. Deaconescu, Transactions of FAMENA, 39, 65 (2015)

5. A. Deaconescu, ICBBE'17, Decision Support System Based on Robust Design Methods, (Seoul, South Korea, 2017) 